\title{
Research
}

Bruce Mason, Kirsty Boyd, John Steyn, Marilyn Kendall, Stella Macpherson and Scott A Murray

\section{Computer screening for palliative care needs in primary care:}

a mixed-methods study

\begin{abstract}
Background

Though the majority of people could benefit from palliative care before they die, most do not receive this approach, especially those with multimorbidity and frailty. GPs find it difficult to identify such patients.
\end{abstract}

\section{Aim}

To refine and evaluate the utility of a computer application (AnticiPal) to help primary care teams screen their registered patients for people who could benefit from palliative care.

\section{Design and setting}

A mixed-methods study of eight GP practices in Scotland, conducted in 2016-2017.

\section{Method}

After a search development cycle the authors adopted a mixed-methods approach combining analysis of the number of people identified by the search with qualitative observations of the computer search as used by primary care teams, and interviews with professionals and patients.

\section{Results}

The search identified $0.8 \%$ of 62708 registered patients. A total of 27 multidisciplinary meetings were observed, and eight GPs and 10 patients were interviewed. GPs thought the search identified many unrecognised patients with advanced multimorbidity and frailty, but were concerned about workload implications of assessment and care planning. Patients and carers endorsed the value of proactive identification of people with advanced illness.

\section{Conclusion}

GP practices can use computer searching to generate lists of patients for review and care planning. The challenges of starting a conversation about the future remain. However, most patients regard key components of palliative care (proactive planning, including sharing information with urgent care services) as important. Screening for people with deteriorating health at risk from unplanned care is a current focus for quality improvement and should not be limited by labelling it solely as 'palliative care'.

\section{Keywords}

advance care planning; anticipatory care; computer searching; general practice; multimorbidity; palliative care; screening

\section{INTRODUCTION}

More than $70 \%$ of people would benefit from a palliative care approach following diagnosis of an advanced progressive illness and during most of their last year of life, ${ }^{1}$ but many people still die having received palliative care only in the last few weeks of life, or not at all. People with multimorbidity and organ failure are particularly disadvantaged, as are frail people living at home. 1,2 Primary care teams adopting a palliative care approach could ensure that more patients' preferences for treatment and care are understood, plans for deterioration are in place, and quality of life is optimised. This would result in less treatment of low benefit, fewer hospital admissions, and more people dying in their place of choice. ${ }^{3,4}$

Palliative care is appropriate for people with any life-limiting condition, and should be integrated with diseasemodifying treatments as soon as possible following diagnosis. ${ }^{5}$ However, healthcare professionals and the general public tend to associate palliative care with terminal illness and imminent death. In response, some palliative care specialists have called for a rebranding of palliative care so that it is more acceptable to all involved. In Scotland, the term anticipatory care planning' (ACP) has recently been adopted

B Mason, PhD, research fellow; K Boyd, PhD, FRCP(Ed), honorary clinical senior lecturer; M Kendall, PhD, research fellow; SA Murray MD, FRCP(Ed), FRCGP, St Columba's Hospice professor of primary palliative care, Primary

Palliative Care Research Group, Usher Institute of Population Health Sciences and Informatics, University of Edinburgh Medical School, Edinburgh. J Steyn, FRCGP, clinical eHealth adviser, NHS Lothian, Edinburgh. S Macpherson, Dip OT, public representative, Primary Palliative Care Research Group, Usher Institute of Population Health Sciences and Informatics, University of Edinburgh, Edinburgh. Address for correspondence Bruce Mason, Primary Palliative Care Research to refer to proactive care planning with people who have one or more serious longterm condition. ${ }^{7}$ This has been implemented nationally, supported by an electronic record - the Key Information Summary (KIS) - that is shared among health service providers with the patient's consent (Box 1).

There is already evidence that having a documented care plan as part of a KIS increases the likelihood of a person dying in the community. ${ }^{8}$ However, patients must be identified more systematically by primary care teams so that anticipatory care and a KIS can be triggered. In 2011, only $29 \%$ of patients who died in Scotland were on a practice palliative care register at death, and $68 \%$ of these had cancer. Only $30 \%$ of deaths that year were from cancer, so most people who died from other conditions were not identified for palliative care before death. ${ }^{9} \mathrm{~A}$ more recent study indicated that anticipatory care has greatly increased the number of patients receiving care planning. ${ }^{8}$ Practices might benefit from practical guidance and tools to support systematic patient identification, whether for anticipatory or palliative care, so that more people with deteriorating health receive holistic care and support.

Tools such as the Supportive and Palliative Care Indicator Tool (SPICTTM), and the Gold Standards Framework Proactive Indicator

Group, Usher Institute of Population Health Sciences and Informatics, University of Edinburgh Medical School, Teviot Place, Edinburgh EH8 9AG, UK.

Email: Bruce.Masonded.ac.uk

Submitted: 25 September 2017; Editor's response: 5 December 2017; final acceptance: 14 December 2017

\section{(CBritish Journal of General Practice}

This is the full-length article (published online $27 \mathrm{Mar} 2018$ ) of an abridged version published in print. Cite this version as: $\mathbf{B r} \mathbf{J}$ Gen Pract 2018; DOl: https://doi.org/10.3399/bjgp18X695729 


\section{How this fits in}

GPs find timely identification and introduction of a palliative care approach challenging in a diverse population with advanced conditions. The tool the authors developed enabled routine data in GP computer databases to be searched successfully, and it identified about 0.8\% of registered patients for care planning reviews. Framing early palliative care as anticipatory care planning (ACP) with patients enabled GPs to provide proactive. multidimensional care consistent with a palliative care approach, without having to confront the stigma often associated with palliative care. Primary care record searching can supplement other casefinding approaches to trigger early palliative care, and the AnticiPal app with guidelines is available for use by practices throughout the UK.

Guidance (GSF PIG) can help GPs trigger palliative care by considering individual cases. ${ }^{10,11}$ The authors previously tested the feasibility of identifying patients using an electronic record search of GP lists based on the SPICT criteria. $^{12}$ That search examined Read Codes (Box 2) stored in a sample of GP databases, and GPs found this useful in identifying potential patients for review. In the present study, the authors aimed to refine and test the utility of GP practices running their own Read-code-based computer searches regularly to help them identify patients for a palliative care approach.

\section{METHOD}

The authors employed an iterative software development life cycle method (Figure 1) to create a more robust application (AnticiPal), based on the search algorithm they had previously developed. ${ }^{12}$ AnticiPal uses a list of Read Codes that singly or in combination might indicate a person

\section{Box 1. Key information summary (KIS)}

The Key Information Summary (KIS) is an IT development in NHS Scotland, pioneering a shared medical record between healthcare professionals. It allows selected parts of the GP electronic patient record to be shared with other parts of the NHS, using a template within the GP clinical system, and is more efficient and safe than previous paper-based and email-based methods. The level of detail contained on a KIS will depend on the complexity of the patient's clinical condition, and it is designed to be added to as the patient's clinical condition progresses. It was introduced in Scotland in 2013, and is an extension to the Emergency Care Summary (ECS). The KIS can contain Read-coded data and free text. Changes to the KIS entered by the patient's general practice are updated to the central KIS every 2 hours. The central KIS can be accessed by out-of-hours and some other services, for example, accident and emergency, an acute receiving unit, and the Scottish Ambulance Service. Although other services can read a KIS, only general practices can lat the time the project was conducted) add information into a KIS.

For more information see http://www.scimp.scot.nhs.uk/key-information-summary/. has deteriorating health and palliative care needs. It specifically excludes people who are already on the practice palliative care register, and also those who are living in a care home, where identification tends to be by care setting (Appendix 1).

The authors recruited eight practices in a Scottish region. Many practices were keen to participate, which allowed the authors to sample urban and rural practices with a spectrum of deprivation indicators. Each practice was considered as a case study in which the authors supported the practice to run the AnticiPal program periodically as part of their routine activities. The authors observed up to six multidisciplinary meetings where the search results were discussed. They interviewed the GP palliative care lead to understand the acceptability and impact on practice. Finally, they interviewed up to two patients from each practice who had been identified for anticipatory or palliative care, to understand their perspectives.

The authors conducted a mixed-methods evaluation of how the search was used over a 6-month period during 2016-2017. An initial version of the search utility was created and used on the patient lists of two practices, who then checked the results against their clinical knowledge. Version 1 was then released to the participating practices. Qualitative feedback from observing meetings in which the application's output was integrated into the normal discussion and/or case finding for patients with palliative care needs was used to inform a rapid software development cycle, ${ }^{13}$ in order to produce a second version that underwent the same cycle.

Anonymised statistics were collected showing the total number of patients identified by AnticiPal each time it was run, which Read Codes had triggered selection, how many patients were newly identified by each screening run, and how many patients were new cases. From this, the authors could also calculate how many patients had been removed between uses of the application.

Up to two patients were recruited from each practice in consultation with the lead GP for palliative care at the practice. Registered patients who were on the practice's palliative care register, or who had given consent for their KIS to be shared with the NHS, were approached and asked to take part in an interview focusing on their understanding of the care they received. They were also asked whether they would be willing for the authors to interview the non-NHS person who was most important in helping manage their care - their main 


\section{Box 2. Read Codes and technical limitations}

Read Codes are a standard clinical vocabulary for use in both primary and secondary care. They exist within a hierarchical thesaurus so that, for example, all codes indicating some form of malignancy are grouped together. GP clinical software can then search Read Codes associated with one or more patients on the practice's registered list to provide information - for example, how many patients $>75$ years have some form of kidney disease.

AnticiPal expands this functionality to perform a series of searches. Because the program uses the preexisting functionality of the current clinical software, there are certain things it cannot search. Most notably, it cannot search for free-text entries. It also cannot search for the presence or content of other documents stored on the system, such as discharge letters or communications from other health professionals.

AnticiPal is also limited by the user interface of the clinical software. Essentially, it can only output information that the clinical software allows. This meant that the authors were restricted to outputting a simple list of matched patients, with no indication of why they were matched. In practice, this had certain advantages, but most participants indicated that they would prefer more detail.

Read Codes are to be retired in 2018 and replaced with a thesaurus known as SNOMED CT (http://www snomed.org//. The principles behind AnticiPal can easily be translated into SNOMED CT.
Figure 1. Software development life cycle.

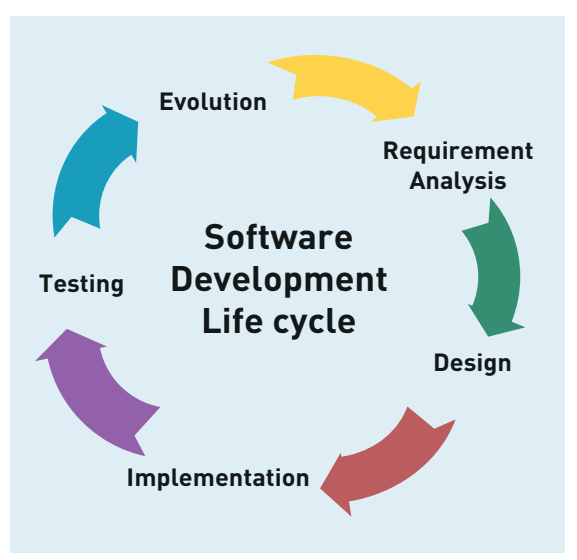

informal carer, for example, a spouse or child. This person was also approached to take part in an interview. In all cases, where a main informal carer took part, they chose to do so in a joint interview with the patient.

Interviews were transcribed and anonymised, with each participant being replaced by a code that indicated sex, role (patient, carer, GP, and so on), and 5-year age range (for example, 65-70 years). Once anonymised and checked, the transcripts were then entered into data analysis software (NVivo version 8). Notes taken during the practice meetings and researcher reflections were anonymised (participants were replaced with codes such as GP3), and added into NVivo as memos. A thematic analysis was conducted to identify the key issues emerging from the application's use. ${ }^{14}$

\section{RESULTS}

An application version of the search was commissioned from a third-party provider - Bluebay Medical Systems - to enable the search to include combinations of Read Codes (Appendix 2). When initially tested in the two pilot practices, $>200$ patients were identified, with about 35\% being considered broadly appropriate, so the authors moved to further testing.

\section{Testing}

During the testing step it became clear that the first version of the application was identifying too many patients for the practice staff to check as part of routine care. The authors therefore developed a second version of the search that removed some of the single Read Codes (Appendix 3). Version 2 of the application was then tested in the same way over the second half of the project. In total, the search was run 33 times and used at multiple meetings ( $n=16$ for version 1, and $n=17$ for version 2). Each practice ran each version at least once. Table 1 shows the results of all runs. The refinement of the search in version 2 produced more manageable list sizes, and identified $0.61 \%$ to $1.23 \%$ of a practice list. The search identified $0.8 \%$ of 62708 registered patients.

During testing of version 2, the authors received feedback that the participants would like to be able to see which of the matched patients already had a KIS. Technological constraints made this impossible to automate, but the research team simulated this function by filtering the results for each practice.

In version 1, about one-third of the patients identified by the search did not already have a KIS, but were considered as likely to benefit from having a KIS or anticipatory care plan by the practice team. In subsequent meetings, the proportion of identified patients without a KIS fell, partly due to a KIS having been completed following a previous search run. Though the researcher did observe some meetings in which a patient was added to the palliative care register, the commonest actions taken were to start or review a KIS. The practice teams found the search most useful in case-finding people for anticipatory care planning rather than palliative care.

\section{Qualitative results}

Eight GPs (professionals) and 10 patients accompanied by an informal carer (service users) were interviewed. A total of 27 multidisciplinary meetings were observed. The primary themes for professionals focused on the practicalities of running the searches and generating lists, identification of more people with non-cancer illnesses, and the notion of a 'pre-palliative' status. For service users, the primary themes centred on being able to maintain an independent lifestyle, perceptions of what the NHS could offer them, and a lack of understanding about palliative care as a concept, while at the same time supporting specific elements of a palliative care approach that they had experienced.

\section{Professional theme: practicalities}

Primary care teams struggled to manage their current workloads and were unsure about how they would provide additional anticipatory care or palliative care for more patients. Professionals felt lack of time might limit their ability to give what they 
Table 1. Summary of patients identified for AnticiPal versions 1 and $2^{\mathrm{a}}$

\begin{tabular}{lccccccccc} 
Patients identified & IP1 & IP2 & IP3 & IP4 & IP5 & IP6 & IP7 & IP8 & Total \\
\hline Version 1 & 462 & 111 & 342 & 332 & 429 & 205 & 333 & 245 & 2459 \\
\hline Version 2 & 55 & 29 & 63 & 69 & 78 & 41 & 106 & 71 & 512 \\
\hline List size & 4459 & 3086 & 9971 & 10832 & 9367 & 6766 & 10847 & 7380 & 62708 \\
\hline V2 by list size & $1.23 \%$ & $0.94 \%$ & $0.63 \%$ & $0.64 \%$ & $0.83 \%$ & $0.61 \%$ & $0.98 \%$ & $0.96 \%$ & $0.82 \%$
\end{tabular}

IP 1-8 is the practice identifier. Version 1 is the number of patients identified by the first version of the search. Version 2 is the number of patients identified by the revised search. List size is the number of patients registered with the practice at the start of the project. V2 by list size refers to the percentage of patients registered at the practice at the start of the project who are identified by the search. considered to be good palliative care to larger numbers of patients:

I'm just thinking, actually, about the number that are on the palliative care register. Now, I think for the palliative care register to mean something it has to be limited. If you have 30 people on the palliative care register, you're not really going to give each patient the same ... I think it's quality rather than quantity.' (GP interview \#2)

\section{Professional theme: identification of all who are dying}

The participants felt that their ability to identify people in need of palliative care for conditions other than cancer had increased, but needed further improvement:

I think we've got a robust system for identifying people for care planning needs, but there are always people that take you by surprise. I think we've still only got this sort of information for about seventy or eighty per cent of our patients that are dying. There's obviously still people who do die without us having given this much thought. (GP interview \#1)

\section{Professional theme: pre-palliative phase}

The computer search provided a list of unstable or frail patients who were suitable for anticipatory care. These patients were depicted as pre-palliative - that is, they would come to need palliative care at some point in the foreseeable future:

I think [a pre-palliative list] is the way forward for us, because I think it might lower people's threshold for thinking about someone in a palliative sense, without having to broach it with the patient themselves.' (GP interview \#8)

Being able to identify patients as prepalliative but at risk, and start a process of anticipatory care planning, enabled professionals to mitigate their concerns relating to conversations about palliative care:

'One patient I know, she is palliative, but I haven't put her on the list and the reason is she's so far from considering herself palliative that, that it would be very upsetting for her.' (GP interview \#4)

\section{Service user theme: independence}

For service users, the focus was primarily on independence and 'living in the present'. There was resistance to planning for the future beyond finance and property concerns, but evidence that once these aspects were completed people thought all the important planning was 'sorted':

We just take every day as it comes. Everything's, financially and everything's all sorted, and it's a horrible thing to do, but when mum passed away I got power of attorney for dad, sorted all the finances out, the house is in my name etc., etc., etc. That's all done. '(Carer interview \#6)

\section{Service user theme: NHS resources}

Though independence was viewed as a positive attribute by many patients and carers, there was also a widely shared belief that GPs were too busy to have conversations about planning for the future, or that they focused primarily on treating medical problems. In part, service users relied on their own resources because they felt they had to:

'Basically, doctors don't have time for all that. You never get a conversation out of a doctor. It's just they come in, they deal with you and they're out again, and that's it. They've not got time to do anything else, and I'm not in the habit of going and making an appointment to sit and discuss things that might happen. They're busy. It's too busy.' (Carer interview \#3)

\section{Service user theme: lack of} understanding about palliative care

Interviews were conducted using a semi-structured method, and relied on using participants' own language rather than imposed medical terms. It was inappropriate to ask participants about palliative care unless the participant used the term first, something that none of them did. When asked in the abstract about whether they had heard of palliative care, and what they knew about it, there was little evidence of any understanding. Certainly, no one volunteered that they were on a 
palliative care register. No one appeared to think that palliative care related to them or to the person they cared for.

In actual fact, there was plenty of evidence that these service users were receiving enhanced care from their local GP practices. For example, one carer talked about how he liked it that his GP always called back straight away when he phoned about his father, and noted that was different from when he contacted the practice about his own health issues. Patients and carers seemed to appreciate such proactive care, but did not regard it as being part of palliative care or any kind of enhanced care from their GP beyond what seemed normal.

\section{DISCUSSION}

\section{Summary}

Systematic screening of patients for palliative care needs by computer searching in primary care can increase the number of people identified for consideration of a palliative care approach. Using this computer search, GPs can produce a list of around $1 \%$ of their practice population who are candidates for an anticipatory or palliative care approach. This can supplement case-finding during routine clinical practice.

However, many patients living with advanced, progressive illnesses and their carers still have little understanding of the nature and potential benefits of palliative care, as defined in national and international policies, associating it with dying and not seeing it as applicable to them. A known barrier to earlier palliative care has been GP concerns about having to start talking about dying too soon. ${ }^{15}$ Screening for people with advanced illnesses, for the purpose of starting either anticipatory or palliative care planning, allowed GPs to start a process with immediate benefits for patient care lsuch as sharing information with urgent care services) that could be gradually built on over time into more formal palliative care.

Screening for people with deteriorating health at risk from unplanned care is a current focus for quality improvement and should not be limited by labelling it solely as palliative care. In Scotland, anticipatory care is now the main approach to proactive care planning for a wide range of people with unstable or deteriorating health and complex care needs. It includes early palliative care, and is acceptable to patients, family carers, and health professionals in the community and hospitals.

In addition, because the search focused on indicators of declining function and unmet health and care needs, it identified many patients with frailty or multimorbidity. Primary care teams were concerned about the resources needed to provide the more intensive care associated with being on palliative care registers for a greater number of patients. Service users were keen to retain independence and control over the aspects of future planning they considered to be important, but appreciated the potential benefits of better coordinated and more readily accessible care from their GP practice.

\section{Strengths and limitations}

Using an iterative software development life cycle model enabled the authors to work with GPs to adapt the application to their needs. The primary care teams were highly engaged in the development, because they could see the advantages it offered. An example of this was the development of an output that let GPs easily check which patients on the list already had a KIS in place. The screening process was conducted in a nationally replicable way without imposing any data input standardisations in the practices, which aids generalisability. However, some potentially useful triggers for palliative care, such as unplanned hospital admissions, are not routinely coded in many practices, so these could not be included in the searches. The regional clinical ehealth lead for primary care was a co-researcher, facilitating an understanding of local and national primary care issues and dissemination.

Working with anonymised data meant that the authors could not document the sensitivity and specificity of the application. Similarly, because the researcher was blinded to patient information it was not possible to evaluate exactly how many patients received additional care, either directly or indirectly, because of identification by the search. There were also limitations around the variation and completeness of data coding and access in GP databases, and the interface for displaying information without a fundamental reprogramming of the clinical software.

\section{Comparison with existing literature}

Identification of people for palliative care remains a major challenge, despite a decade of initiatives. ${ }^{16}$ In Europe, various systems are being developed to identify patients for palliative care more systematically. ${ }^{17}$ Similar approaches are also being taken with related cohorts, such as the development of an Electronic Frailty Index, ${ }^{18}$ and an 


\section{Funding}

This work was supported by Marie Curie, Edinburgh and Lothians Foundation, and the OAK Foundation as part of a Lothian Palliative Care redesign project.

\section{Ethical approval}

This study was reviewed by the South East Scotland Research Ethics Committee 2. In order to facilitate the iterative program development, the authors took advice to not access patient-identifiable data while evaluating the program's results. This enabled them to develop the program as part of a service evaluation. Consequently, the research was proportionately reviewed by the chair and liaison, and it was not judged necessary to conduct a full review (Ref: NR/1503AB8).

\section{Provenance}

Freely submitted; externally peer reviewed.

\section{Competing interests}

The authors have declared no competing interests.

\section{Open access}

This article is Open Access: CC BY-NC 4.0 licence (http://creativecommons.org/ licences/by-nc/4.0/).

\section{Acknowledgements}

The authors wish to thank all practice staff, patients, and their carers who participated. They also thank the eHealth GMS Facilitators at NHS Lothian for their help in developing AnticiPal.

\section{Discuss this article}

Contribute and read comments about this article: bjgp.org/letters

interest in understanding and measuring increasing dependency in later life. ${ }^{19}$ In practice, AnticiPal proved particularly good at identifying patients with multimorbidity - an underserved population. ${ }^{20}$ Descriptive tools, such as the SPICTTM, ${ }^{10}$ could also be used as part of a systematic screening, and an app is available, and an electronic version of the GSF PIG is being developed. ${ }^{21}$

There is increasing interest in the use of clinical data to provide benefits at the individual patient level, as well as aggregation of data for public health information. ${ }^{22}$ AnticiPal shows how clinical data that is routinely collected in primary care can be used to help identify individual patients for planning and care coordination. Currently, many uses of data held in primary care (for example, risk of re-admission lists) is exported to a remote facility, analysed with admissions data, then returned to GPs. This means such data risks being out of date before it is used. A run-on demand application that searches the current data held within its own system enables practices to use it in the ways that best fit their needs, such as in advance of multidisciplinary team meetings, or as part of local primary care-led quality improvement initiatives.

This approach fits within the literature associated with the concept of 'realistic medicine. ${ }^{23,24}$ The idea is to reduce overmedicalisation, unwarranted variation, and interventions of low benefit, replacing these with a focus on maximising quality of life. This approach is based on health professionals working in conjunction with patients to direct their own care. ${ }^{24}$ This corresponds with the qualitative findings from the authors' research that found patients also seek to minimise the medicalisation of their life, while receiving appropriate support for shared decision making. AnticiPal is not a search designed to identify patients who need more medical input, but to flag up people who may benefit from proactive planning to improve quality of life.

\section{Implications for practice}

Using AnticiPal or a similar search tool will identify more people for what is essentially a palliative care approach, and can help trigger care planning and coordination. It may be better to refer to this approach using a name that is acceptable to patients and their carers, such as anticipatory care, which is now being widely adopted for a broad range of people with complex health and care needs in Scotland. The stigma associated with receiving palliative care, and the difficulties of talking about dying in concrete terms before people are ready, would not then continue to prevent people benefiting from the holistic care they need. GPs still face the challenge of starting conversations about the future, but there are evidence-based approaches that allow for uncertainty, and help people to consider what they might like or would not want 'if' instead of 'when' they become more unwell. ${ }^{2}$ This approach does have resource implications in primary care. Proactively searching in this way may reduce the number of crises that occur in primary care, due to appropriate care and support, and reduce inappropriate interventions. 


\section{REFERENCES}

1. Murtagh FE, Bausewein C, Verne J, et al. How many people need palliative care? A study developing and comparing methods for population-based estimates. Palliat Med 2014; 28(1): 49-58.

2. Kimbell B, Murray SA, Macpherson S, Boyd K. Embracing inherent uncertainty in advanced illness. BMJ 2016; 354: i3802.

3. Murray SA, Kendall M, Mitchell G, et al. Palliative care from diagnosis to death. BMJ 2017; 356: j878

4. Mitchell G, Senior HE, Rhee JJ, et al. Using intuition or a formal palliative care needs assessment screening process in general practice to predict death within 12 months: a randomised controlled trial. Palliat Med 2017; DOI: 10.1177/0269216317698621.

5. Connor SR, Sepulveda Bermedo MC. Global atlas of palliative care at the end of life 2014. World Health Organization and Worldwide Palliative Care Alliance, 2014. http://www.who.int/nmh/Global_Atlas_of_Palliative_Care.pdf laccessed 30 Jan 2018).

6. Zimmermann C, Swami N, Krzyzanowska M, et al. Perceptions of palliative care among patients with advanced cancer and their caregivers. CMAJ 2016: 188(10): E217-E227.

7. Healthcare Improvement Scotland. Anticipatory care planning toolkit 2017 http://ihub.scot/anticipatory-care-planning-toolkit/ (accessed 30 Jan 2018).

8. Tapsfield J, Hall C, Lunan C, et al. Many people in Scotland now benefit from anticipatory care before they die: an after death analysis and interviews with general practitioners. BMJ Support Palliat Care 2016; DOI: 10.1136/ bmjspcare-2015-001014.

9. Harrison N, Cavers D, Campbell C, Murray SA. Are UK primary care teams formally identifying patients for palliative care before they die? Br J Gen Pract 2012; https://doi.org/10.3399/bjgp12X641465

10. Highet G, Crawford D, Murray SA, Boyd K. Development and evaluation of the Supportive and Palliative Care Indicators Tool (SPICT): a mixed-methods study. BMJ Support Palliat Care 2014; 4(3): 285-290.

11. Maas EAT, Murray SA, Engels $Y$, Campbell C. What tools are available to identify patients with palliative care needs in primary care: a systematic literature review and survey of European practice. BMJ Support Palliat Care 2013; 3(4): 444-451.

12. Mason B, Boyd K, Murray SA, et al. Developing a computerised search to help
UK general practices identify more patients for palliative care planning: a feasibility study. BMC Fam Pract 2015; 16(1): 99.

13. Haag S, Cummings M, McCubbrey DJ. Management information systems for the information age. 3rd edn. Toronto: McGraw-Hill Ryerson, 2006.

14. Pope C, Ziebland S, Mays N. Analysing qualitative data. BMJ 2000; 320: 114-116.

15. Boyd K, Mason B, Kendall M, et al. Advance care planning for cancer patients in primary care: a feasibility study. Br J Gen Pract 2010; DOI: https://doi. org/10.3399/bjgp10X544032.

16. Murray SA, Sheikh A. Care for all at the end of life. BMJ 2008; 336(7650): 958-959.

17. Gómez-Batiste X, Murray SA, Thomas K, et al. Comprehensive and integrated palliative care for people with advanced chronic conditions: an update from several European initiatives and recommendations for policy. J Pain Symptom Manage 2017; 53(3): 509-517.

18. Clegg A, Young J, Iliffe S, et al. Frailty in elderly people. Lancet 2013; 381(9868): 752-762.

19. Kingston $A$, Wohland $P$, Wittenberg $R$, et al. Is late-life dependency increasing or not? A comparison of the Cognitive Function and Ageing Studies (CFAS). Lancet 2017; 390: 1676-1684.

20. Mason B, Nanton V, Epiphaniou E, et al. 'My body's falling apart.' Understanding the experiences of patients with advanced multimorbidity to improve care: serial interviews with patients and carers. BMJ Support Palliat Care 2016; 6: 60-65.

21. Gold Standards Framework. PIG - Proactive Identification Guidance Registration Form. http://www.goldstandardsframework.org.uk/pig laccessed 20 Mar 2018).

22. Ibrahim $\mathrm{K}$, Owen $\mathrm{C}$, Patel HP, et al. Can routine clinical data identify older patients at risk of poor healthcare outcomes on admission to hospital? BMC Res Notes 2017; 10(1): 384

23. Calderwood C. Realising realistic medicine. The Chief Medical Officer for Scotland's Annual Report 2015/16. Edinburgh: Scottish Government, 2017 https://beta.gov.scot/publications/chief-medical-officer-scotland-annualreport-2015-16-realising-realistic-9781786526731/pages/9/ laccessed 30 Jan 2018).

24. Christie B. Catherine Calderwood: champion of 'realistic medicine'. BMJ 2016; 355: i5455. 


\section{Appendix 1. AnticiPal utility specification}

The AnticiPal data extract module searches for patients with one or more indicators of palliative care needs who are NOT currently on the palliative care register and who are NOT currently living in a care home.

\section{Summary}

AnticiPal runs three different types of search.

1. Read Codes that indicate a malignancy that has been entered within the last 18 months. If the code was entered more than 18 months ago, and there has been no repetition of the code since then, the patient is considered likely to be stable or in remission.

2. Single Read Codes that are often associated with indicators that a person would benefit from anticipatory or palliative care. This includes codes such as $2 \mathrm{Jd}$ ('frailty'). Note that there are two codes that explicitly reference palliative care: Z172 and 8BAN. These two codes are not in the listing of Read Codes that are automatically included in the palliative care register.

3. Combination searches. There are seven different combinations of Read Codes that can trigger an identification.

AnticiPal then excludes anyone who is already on a palliative care register or who is living in a care home. It does this by checking for any Read Code that, if present, would automatically add the patient to the practice palliative care register. For example, if the code 1Z01 ('terminal illness - late stage') is present, that patient will have automatically been added to the practice's palliative care register. This patient is already identified, therefore AnticiPal does not include them in the list of patients.

If at least one of the three types of search succeeds and the patient is not excluded, AnticiPal identifies the patient as possibly benefiting from anticipatory or palliative care.

\section{Appendix 2. AnticiPal search algorithm specification}

Patient is included if one or more inclusion criteria are met, AND NO exclusion criteria are met.

\section{Inclusion criteria}

\section{Single Read Codes}

Type 1 - Malignancy within 18 months

\begin{tabular}{|c|c|c|c|}
\hline Type & Code & Title & Time frame \\
\hline 1 & $\mathrm{~B} 11 \%$ & Malignant neoplasm of stomach & $\leq 18$ months \\
\hline 1 & $\mathrm{~B} 17 \%$ & Malignant neoplasm of pancreas & $\leq 18$ months \\
\hline 1 & $\mathrm{~B} 22 \mathrm{z}$ & Malignant neoplasm of bronchus or lung & $\leq 18$ months \\
\hline 1 & $\mathrm{~B} 56 \%$ & Secondary and unspecified malignant neoplasm of lymph nodes & $\leq 18$ months \\
\hline 1 & $\mathrm{~B} 57 \%$ & Secondary malignant neoplasm of respiratory and digestive systems & $\leq 18$ months \\
\hline 1 & $\mathrm{~B} 58 \%$ & Secondary malignant neoplasm of other specified sites & $\leq 18$ months \\
\hline 1 & BB03 \% & Neoplasm, metastatic & $\leq 18$ months \\
\hline 1 & BB13. & [M] Carcinoma, metastatic, NOS & $\leq 18$ months \\
\hline 1 & BB14. & [M] Carcinomatosis & $\leq 18$ months \\
\hline 1 & 8BAD & Chemotherapy & $\leq 18$ months \\
\hline 1 & $7 \mathrm{M} 371$ & Radiotherapy NEC & $\leq 18$ months \\
\hline \multicolumn{4}{|c|}{ Type 2 - Other single Read Codes } \\
\hline 2 & $\mathrm{Z172}$ & Palliative care & \\
\hline 2 & $1 \mathrm{D} 18$ & Pain from metastases & \\
\hline 2 & 6639 & Oxygen at home & \\
\hline 2 & $66 Y_{j}$ & Home oxygen supply cylinder & \\
\hline 2 & $66 \mathrm{Yk}$ & Home oxygen supply concentrator & \\
\hline 2 & 877 & Oxygen therapy & \\
\hline 2 & 8776 & Long-term oxygen therapy & \\
\hline 2 & 8CAX & Recommended thickened fluids & \\
\hline 2 & 8BAN & Community specialist palliative care & \\
\hline 2 & $13 C D$ & Mobility very poor & \\
\hline 2 & $\mathrm{H} 39$ & Very severe COPD & \\
\hline
\end{tabular}




\section{Appendix 2 continued. AnticiPal search algorithm specification}

\begin{tabular}{lll}
2 & 6991 & Geriatric home admission exam \\
\hline 2 & $\mathrm{C} 19$ & Multiple organ failure \\
\hline 2 & $2 \mathrm{Jd}$ & Frailty \\
\hline 2 & 1688 & Exhaustion \\
\hline 2 & $\mathrm{~K} 055$ & Chronic kidney disease stage 5 \\
\hline 2 & $\mathrm{R} 095 \%$ & Ascites
\end{tabular}

$C O P D=$ chronic obstructive pulmonary disease. $N E C=$ neuroendocrine carcinoma. NOS $=$ Not otherwise specified

Combinations of Read Codes (type 3)

1. Liver disease AND Spironolactone (any dosage) (within 3 months) acute or repeat

a. J61\% (not J610, J611, J616, J61y) AND spironolactone (within 3 months) acute or repeat

2. Fractured neck of femur $\mathrm{S} 30 \%$ within last 12 months $\mathrm{AND}$ age $>80$.

3. Heart failure AND (CKD 4 OR CKD 5) AND COPD.

a. G58\% AND (1Z13 or K054 or 1 Z14 or K055) AND (H3; or H31\% excl H3101 or H31yO or H3122; or H32\%; or H36-H3z (excl H3yO or H3y1); or H5832

4. Peripheral vascular disease AND (CKD 4 OR CKD 5):

a. (G73z\% or G73) AND (1Z13 or K054 or 1214 or K055)

5. Difficulty swallowing AND dementia:

a. R0720 AND (E00\%; or EU02\%, or EU01\%; or E02y1; or E012\%; or Eu00\%; or E041; or EU041; or F110-F112; or F116)

6. Housebound AND dementia

a. 13CA AND (E00\%; or EU02\%, or EU01\%; or E02Y1; or E012\%; or EU00\%; or E041; or EU041; or F110-F112; or F116)

7. 13CA Housebound AND multimorbidity

\section{Exclusions - any of the following Read Codes}

If any of the following codes are present the patient is excluded from the search output, regardless of how many inclusion codes are matched.

\begin{tabular}{|c|c|}
\hline Palliative & \\
\hline $8 \mathrm{H} 6 \mathrm{~A}$ & Refer to terminal care consult \\
\hline $8 \mathrm{H} 7 \mathrm{~g}$ & Referral to palliative care service \\
\hline 8H7L & Refer for terminal care \\
\hline ZV57C & [V]Palliative care \\
\hline 9367 & Patient-held palliative care record \\
\hline $1 Z 01$ & Terminal illness - late stage \\
\hline 2JE & Last days of life \\
\hline 8BA2 & Supportive care \\
\hline 8BAe & Anticipatory palliative care \\
\hline 8BAP & Specialist palliative care \\
\hline 8BAS & Specialist palliative care treatment - day care \\
\hline 8BAT & Specialist palliative care treatment - outpatient \\
\hline 8BJ1 & Palliative treatment \\
\hline $8 \mathrm{CM} 1 \%$ & On Gold Standards Palliative Care Framework \\
\hline $8 \mathrm{CM} 4$ & Liverpool Care Pathway for the Dying \\
\hline $8 \mathrm{CME}$ & Has end-of-life advanced care plan \\
\hline $8 C M Q$ & On Liverpool Care Pathway for the Dying \\
\hline 8CMW3 & End-of-life care pathway \\
\hline $8 \mathrm{HH7}$ & Refer to community specialist palliative care team \\
\hline 8IEE & Referral to community palliative care team declined \\
\hline 9cOLO & Planned palliative oncology or radiotherapy treatment \\
\hline 9c0M & Planned supportive care for terminal illness \\
\hline $9 \mathrm{CON}$ & Current supportive care for terminal illness \\
\hline 9c0P & Current palliative oncology treatment \\
\hline
\end{tabular}




\section{Appendix 2 continued. AnticiPal search algorithm specification}

\begin{tabular}{ll} 
9EB5 & DS 1500 Disability living allowance (terminal care) completed \\
\hline $9 \mathrm{G8}$ & Ambulance service notified of patient on end-of-life care register \\
\hline $9 \mathrm{~K} 9$ & Palliative care handover form completed \\
\hline $9 \mathrm{Ng} 7$ & On end-of-life care register \\
\hline $9 \mathrm{NgD}$ & Under care of palliative care service \\
\hline $9 \mathrm{NNd}$ & Under care of palliative care specialist nurse \\
\hline Other excluding codes & \\
\hline 13FX & Lives in care home \\
\hline
\end{tabular}

\section{Appendix 3. Changes for version 2}

There were several changes made for version 2. The authors removed several single Read Codes that were over-generating or were tending to be used in ways by one or more of the practices that did not indicate palliative or anticipatory care needs. Two of these (173L. and 1Z14.) were being sampled in the combination searches, so were redundant. The code 'Not For Resuscitation' (1R1..) appeared to be redundant in that it was not identifying any patient not identified elsewhere. The rest were identifying many patients, but not the intended cohort.

\begin{tabular}{ll}
\hline Single Read Codes removed for final version (2) of the search & \\
\hline $13 \mathrm{CA}$ & Housebound \\
\hline $173 \mathrm{C}$. & Short of breath on exertion \\
\hline $173 \mathrm{~L}$. & MRC Breathlessness Scale: grade 5 \\
\hline $1 \mathrm{R} 1 .$. & Not for resuscitation \\
\hline $1 \mathrm{Z14}$. & Chronic kidney disease stage 5 \\
\hline 2127 & Patient condition worsened \\
\hline $918 \mathrm{~F}$. & Has a carer \\
\hline $\mathrm{J} 61 \%$ & Cirrhosis and chronic liver disease \\
\hline
\end{tabular}

